\title{
OV/P1-1 \\ Results of Joint Experiments and other IAEA Activities on Research Using Small Tokamaks
}

$\underline{\text { M Gryaznevich }^{1}}$, G Van Oost ${ }^{2}$, P Peleman $^{2}, \mathrm{~J} \mathrm{Brotankova}^{3}, \mathrm{R}^{3}$ Dejarnac $^{3}$, E Dufkova $^{3}$, I Duran $^{3}$, M Hron $^{3}$, J Sentkerestiova ${ }^{3}$, J Stöckel ${ }^{3}$, V Weinzettl ${ }^{3}$, J Zajac $^{3}$, L A Berni ${ }^{4}$, E Del Bosco ${ }^{4}$, J G Ferreira ${ }^{4}$, F J R Simões ${ }^{4}$, M Berta ${ }^{5}$, ${ }^{7}$, D. Dunaí, B TáP, S Zoletnik ${ }^{5}$, A Malaquias ${ }^{6}$, G Mank $^{6}$, H. Figueiredo ${ }^{7}$, Y Kuznetsov ${ }^{7}$, L Ruchko ${ }^{7}, \mathrm{H} \mathrm{Hegazy}^{8}$, A Ovsyannikov ${ }^{9}$, E Sukhov 9 , G M Vorobjev ${ }^{9}$, D Dreval $^{10}$, A Singh $^{10}$, V Budaev ${ }^{11}$, G Kirnev $^{11}$, N Kirneva ${ }^{11}$, B Kuteev ${ }^{11}$, A Melnikov ${ }^{11}$, D Nurov ${ }^{11}$, M Sokolov ${ }^{11}$, V Vershkov ${ }^{11}$, A Talebitaher ${ }^{12}$, P Khorshid ${ }^{12}$, R Gonzales ${ }^{14}$, I El Chama Neto ${ }^{15}$, A W Kraemer-Flecken ${ }^{16}$, $V_{\text {V Soldatov }}{ }^{16}$, B Brotas ${ }^{17}, \mathrm{P}_{\text {Carvalho }}{ }^{17}$, R Coelho ${ }^{17}$, A Duarte $^{17}, \mathrm{H}$ Fernandes ${ }^{17}$, J. Figueiredo $^{17}$, A Fonseca ${ }^{17}$, R. Gomes ${ }^{17}$, I Nedzelski ${ }^{17}$, A Neto ${ }^{17}$, G. Ramos ${ }^{17}$, J Santos ${ }^{17}$, $\mathrm{C} \mathrm{Silva}^{17}$, D Valcárcel ${ }^{17}$, C R Gutierrez Tapia ${ }^{18}$, L I Krupnik ${ }^{19}$, L Petrov ${ }^{20}$, M Kolokoltsov ${ }^{21}$, J. Herrera ${ }^{22}$, M Nieto-Perez ${ }^{23}$, A Czarnecka ${ }^{24}, \mathrm{P} \mathrm{Balan}^{25}$, A Sharnin ${ }^{26}$, V Pavlov ${ }^{26}$.

${ }^{I}$ EURATOM/UKAEA Fusion Association, Culham SC, Abingdon, UK; ${ }^{2}$ Dep. of Applied Physics, Ghent Univ., Belgium; ${ }^{3}$ Institute of Plasma Physics, Association EURATOM/ IPP.CR, Czech Rep.; ${ }^{4} I N P E$, São José dos Campos, Brazil, ${ }^{5}$ KFKI-RMKI, Association EURATOM, Budapest, Hungary; ${ }^{6}$ IAEA, NAPC Physics Section, Vienna, Austria; ${ }^{7}$ Institute of Physics, Univ. of São Paulo, Brazil; ${ }^{8}$ EAEA, Cairo, Egypt; ${ }^{9}$ Saint Petersburg State Univ., Russia; ${ }^{10}$ Plasma Physics Lab., Univ. of Saskatchewan, Canada; ${ }^{11}$ RRC "Kurchatov Institute”, Moscow, Russia; ${ }^{12}$ Plasma Physics Research Center, Teheran, Iran; ${ }^{13}$ Széchenyi István Univ., Association EURATOM, Gyor, Hungary; ${ }^{14}$ CICATA-IPN, Mexico; ${ }^{15}$ Univ. Tuiuti Paraná, Brazil; ${ }^{16}$ Forschungszentrum, Julich, Germany; ${ }^{17}$ IST, Lisbon, Portugal; ${ }^{18}$ ININ, Mexico; ${ }^{19}$ Phys-Tech Inst., Kharkov, Ukraine, ${ }^{20}$ INRNE, Sofia, Bulgaria, ${ }^{21}$ IAE, NNC, Kurchatov, Kazakhstan; ${ }^{22}$ ICN, UNAM, Mexico, ${ }^{23}$ ITT, Mexico; ${ }^{24}$ Association EURATOM IPPLM, Poland; ${ }^{25}$ Institute of Ionphysics, University of Innsbruck, Austria; ${ }^{26}$ TPU, Tomsk, Russia.

Mikhail.gryaznevich@ukaea.org.uk

\begin{abstract}
This paper presents an overview of the results obtained during the Joint Experiments (JE) organized in the framework of the IAEA Coordinated Research Project (CRP) on "Joint Research Using Small Tokamaks" (JRUST) that have been carried out on the tokamaks CASTOR at IPP Prague, Czech Republic (2005), T-10 at RRC "Kurchatov Institute”, Moscow, Russia (2006) and the most recent one at ISTTOK at IST, Lisbon, Portugal, in 2007.. Experimental programmes were aimed to diagnose and characterize the core and the edge plasma turbulence in a tokamak in order to investigate correlations between the occurrence of transport barriers, improved confinement, electric fields and electrostatic turbulence using advanced diagnostics with high spatial and temporal resolution. On CASTOR and ISTTOK, electric fields were generated by biasing an electrode inserted into the edge plasma and an improvement of the global particle confinement induced by the electrode positive biasing has been observed. Geodesic Acoustic Modes were studied using heavy ion beam diagnostics (HIBD) on T-10 and ISTTOK and correlation reflectometry on T-10. ISTTOK is equipped with gallium jet injector and the technical feasibility of gallium jets interacting with plasmas has been investigated in pulsed and AC operation. The first JEs have clearly demonstrated that small tokamaks are suitable for broad international cooperation to conduct dedicated joint research programmes. Other activities within the IAEA CRP on JRUST are also overviewed.
\end{abstract}

\section{Introduction.}

Small tokamaks continue to play an important role in fusion research. Currently, 39 small tokamaks are operational. A new concept of interactive co-ordinated joint research using small tokamaks in the scope of the IAEA Co-ordinated Research Project "Joint Research Using Small Tokamaks" is a further step in better co-ordination of collaboration 
between small tokamaks and in improvements of links between small and large tokamaks. Since the beginning of the CRP in 2004, it has already resulted in more than 30 joint publications. 13 tokamaks are currently participating in the project and an overview of the present activities can be found in [1] and at www.fusion.org.uk/iaeacrp. One of the most successful activities within the CRP is the implementation of Joint Experiments gathering several experts from the fusion community on a particular device during several experimental sessions lasting about 4 to 5 days. In this paper we summarize the results obtained during Joint Experiments.

Joint Experiments in the framework of the IAEA CRP on JRUST have been carried out on the tokamaks CASTOR at IPP Prague, Czech Republic, T-10 at RRC "Kurchatov Institute", Moscow, Russia and ISTTOK at IST, Lisbon, Portugal, in 2005 -2007. The se Joint Experiments involved more than 65 scientists from 16 countries, coordinated and cosupported by IPP Prague, KFKI-RMKI Budapest, RRC "Kurchatov Institute" Moscow, IST, Lisbon, IAEA and ICTP, Trieste, and were aimed to diagnose and characterize the core and the edge plasma turbulence using advanced diagnostics with high spatial and temporal resolution.

\section{2. $1^{\text {st }}$ Joint (Host Laboratory) Experiment, CASTOR, IPP Prague, Czech Republic, 2005.}

The objective of the $1^{\text {st }} \mathrm{JE}$ on CASTOR $\left(\mathrm{R}=0.4 \mathrm{~m}, \mathrm{a}=0.085 \mathrm{~m}, \mathrm{~B}_{\mathrm{t}}<1.5 \mathrm{~T}, \mathrm{I}_{\mathrm{p}}<25 \mathrm{kA}\right.$, $\left.\tau_{\text {pulse }}<50 \mathrm{~ms}, 0.5<\mathrm{n}_{\mathrm{e}}\left(10^{19} \mathrm{~m}^{-3}\right)<3.0, \mathrm{~T}_{\mathrm{e}}(0)<200 \mathrm{eV}\right)$ was to perform studies of links between the plasma edge turbulence and the plasma confinement [2]. It was jointly organized by the IPP

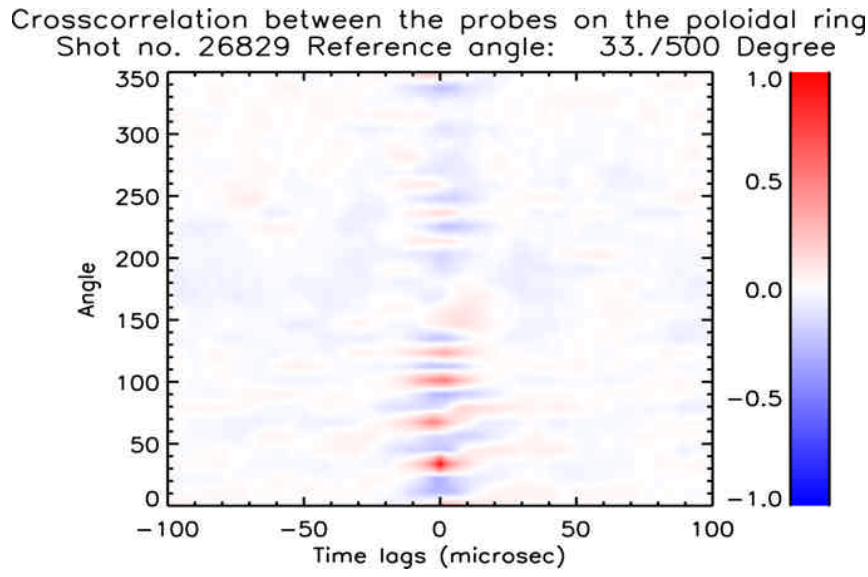

Fig.1. Spatial-temporal correlation function along the poloidal ring of probes.
Prague and KFKI RMKI, Budapest, involved 20 scientists from 7 countries and was supported through the IAEA and the International Centre for Theoretical Physics (ICTP), Trieste. During the experiments, electric fields were generated by biasing an electrode inserted into the edge plasma to modify the turbulence and transport behavior in this region. The edge plasma and the electrostatic turbulence were characterized using two rake probes with 16 Langmuir tips each (radial separation $2.5 \mathrm{~mm}$ ). From the time shift between two poloidally separated tips it was possible to measure the poloidal velocity of fluctuating density and plasma floating potential structures. From the gradient of the floating potential, the phase velocities, $\phi_{f}$, were roughly estimated as $\mathrm{v}=\mathrm{E}_{\mathrm{r}} / \mathrm{B}$, where $\mathrm{E}_{\mathrm{r}}$ is estimated as grad $F_{f}$. However, it was found that the electron temperature gradient grad $\mathrm{T}_{\mathrm{e}}$ term can not be fully neglected, if a precise comparison of the phase and the ExB velocity is required. The phase velocity of potential fluctuations in the poloidal direction was measured using a poloidal array of 96 Langmuir probes arranged uniformly poloidally at one toroidal position. It was found that the phase velocity of density fluctuations was systematically lower than that of potential fluctuations. From the spatial 
temporal behavior of cross-correlation functions of radially separated tips, a radial size of the fluctuating structures of about $1 \mathrm{~cm}$ has been determined. A typical correlation plot is shown in Fig.1. The reference probe was at $\theta=33.75^{\circ}$ relative to the equatorial plane on the LCFS. Spatial wave-like structures (electromagnetic features of turbulence) were observed in the range between $\theta \sim 0^{\circ}$ and $135^{\circ}\left(\theta=0^{\circ}\right.$ corresponds to the outer point at the equatorial plane).

Positive electrode edge biasing experiments have been performed to demonstrate the
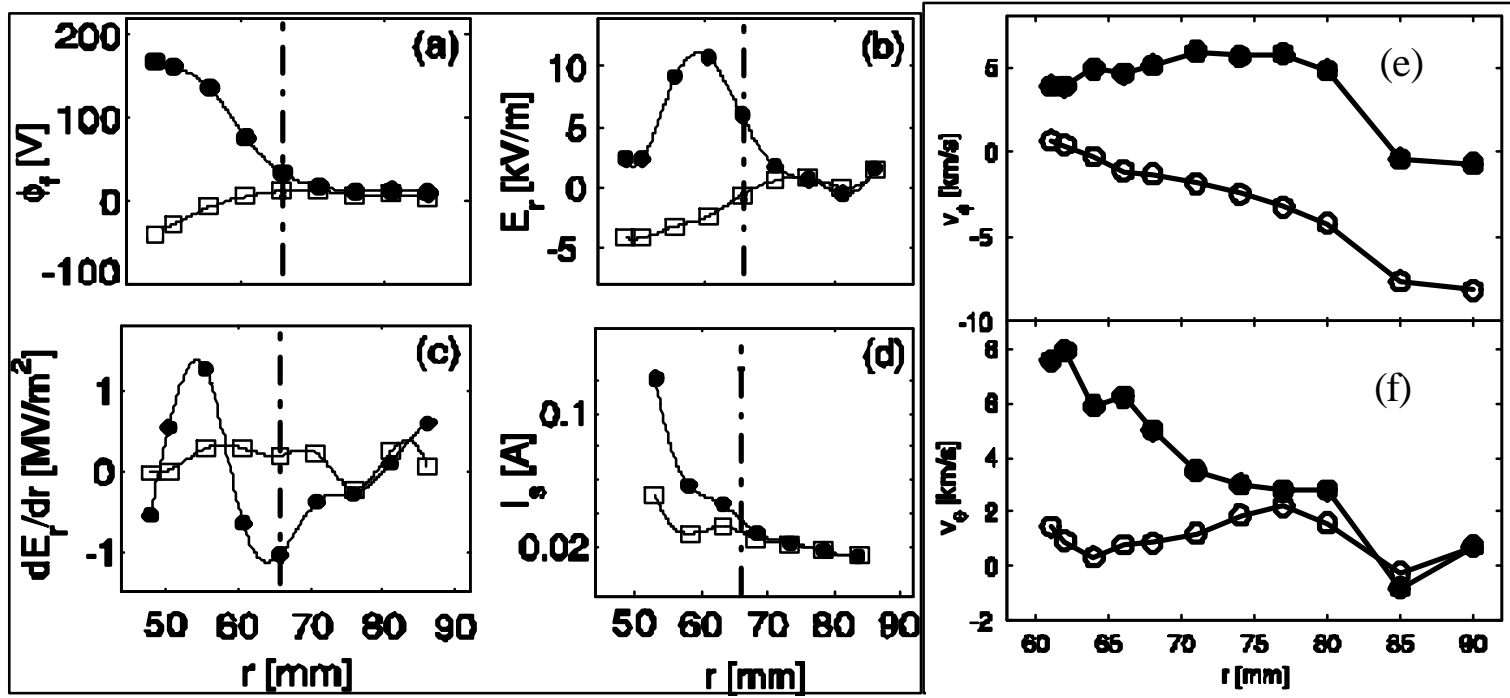

Fig.2. Radial profiles of floating potential (a), radial electric field (b), the $\mathrm{E}_{\mathrm{r}}$ shear, (c) ion saturation current $(d)$, toroidal, $\mathrm{v}_{\phi},(e)$ and poloidal velocity, $\mathrm{v}_{\theta}(f)$, averaged over 4 ms before (open symbols) and during (filled symbols) biasing The vertical line marks the position of the LCFS.

effects of electric fields on the main plasma parameters. Fig. 2 illustrates the influence of positive biasing on the radial dependence of edge plasma parameters. During the biasing phase, the radial dependence of $\phi_{f}$ is strongly modified as shown in Fig.2(a), leading to a narrow positive and single-peaked $\mathrm{E}_{\mathrm{r}}$ structure just inside the LCFS, Fig.2(b). As a consequence, a strong positive and negative $\mathrm{E}_{\mathrm{r}}$ shear is generated inside and across the LCFS, respectively, as shown in Fig.2(c). The maximum shear rate of the $E_{r} \times B$ flow, $\tau_{\mathrm{s}}^{-1} \propto \mathrm{dv}_{\overline{\mathrm{E}} \times \overline{\mathrm{B}}} / \mathrm{dr}$, is thus about $1-1.3 \times 10^{6} \mathrm{~s}^{-1}$. The decorrelation rate of local turbulence scattering, $\tau_{c 0}^{-1}$, calculated from the e-folding time of the autocorrelation function of $\mathrm{I}_{\mathrm{s}}$ fluctuation data detected before biasing, gives $\tau_{c 0}^{-1}=1.6 \times 10^{5} \mathrm{~s}^{-1}$. Hence, the flow shear rate exceeds significantly the turbulence scattering rate and thus suppresses turbulence and turbulent transport. The reduction in $\mathrm{I}_{\mathrm{s}}$ and $\phi_{f}$ fluctuations during biasing has been observed in the experiments. The reduced turbulent transport leads to the formation of an edge pedestal and thus steepening of the edge density profile during biasing, as shown by the ion saturation current measurements in Fig.2(d). During the initial stage of the biasing, a clear reduction in recycling indicated by a drop in the $\mathrm{H}_{\mathrm{a}}$ emission, and thus, a net increase of the ratio $\overline{\mathrm{n}}_{\mathrm{e}} / \mathrm{H}_{\mathrm{a}}$ (which is roughly proportional to the particle confinement time $\mathrm{t}_{\mathrm{p}}$ ) by a factor of 2.5 with respect to the pre-bias phase, has been observed. These results indicate an improvement of the global particle confinement induced by the electrode positive biasing, as observed earlier [3]. Flow measurements have been performed using a Gundestrup probe with 8 collectors. The time evolution of the radial profiles of floating potential, radial electric field, 
parallel and perpendicular Mach numbers were measured in the biased and ohmic phases of a single discharge. The radial flow profiles were measured by a shot-to-shot scan in reproducible discharges. It is found that not only the perpendicular flow, but also the parallel flow increases during biasing, as shown in Fig.2 e,f.

Two arrays of fast AXUV-based bolometers with 16 and 19 channels were installed in the same poloidal cross-section in perpendicular directions (from LFS and bottom side) to monitor the radiated power profile. This arrangement with temporal resolution of $1 \mu \mathrm{s}$ and spatial resolution of $\sim 1 \mathrm{~cm}$ and a very high signal to noise ratio allowed a visualization of the evolution of fine structures in the radiated power profile during biasing. Fig. 3 shows crosscorrelation time evolution between the horizontal chord at $40 \mathrm{~mm}$ against all bottom bolometer array chords prior to biasing (left figure), during biasing with biasing voltage $+150 \mathrm{~V}$ (middle figure) and after biasing (right figure) with a clear change in the correlation length due to biasing, suggesting suppression of small-scale turbulence.

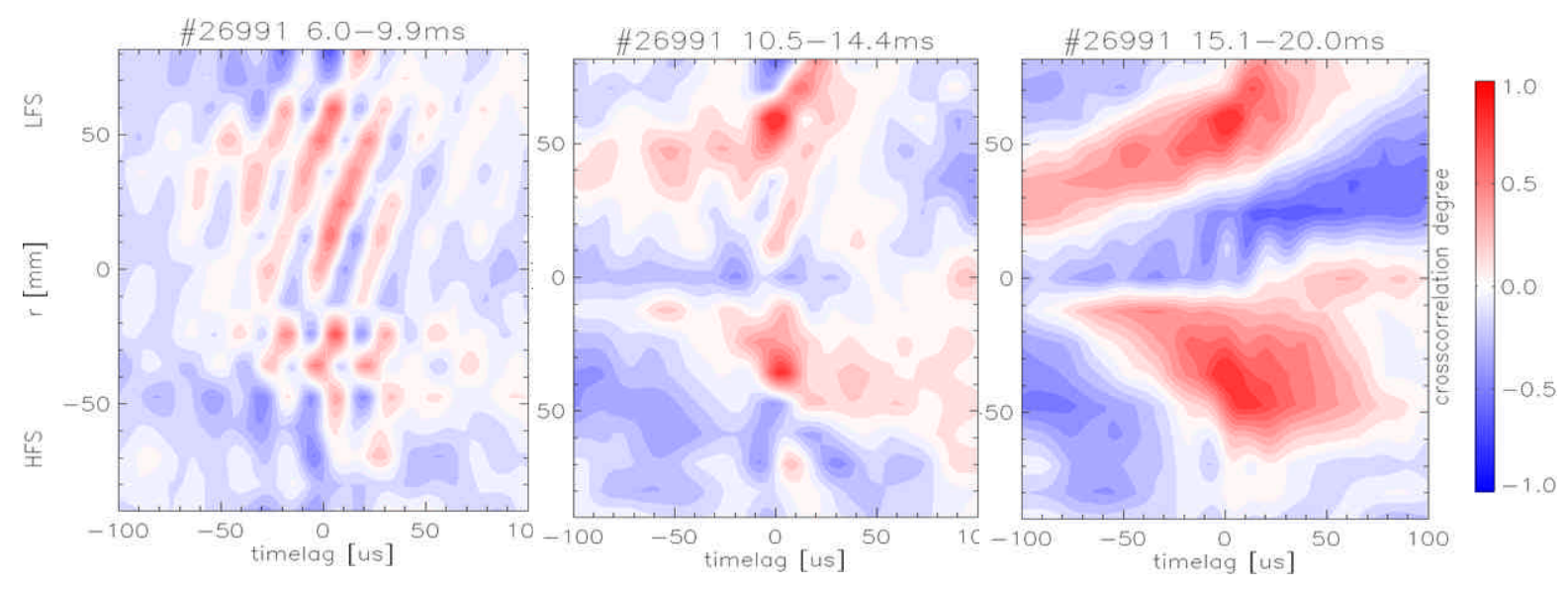

Fig.3. Cross-correlation between horizontal chord at $40 \mathrm{~mm}$ against all bottom chords prior to biasing (left figure), during biasing with biasing voltage $+150 \mathrm{~V}$ (middle figure) and after biasing (right figure).

\section{3. $2^{\text {nd }}$ Joint Experiment, T-10, RRC “Kurchatov Institute”, Moscow, RF, 2006.}

Following the success of the $1^{\text {st }} \mathrm{JE}$, the $2^{\text {nd }} \mathrm{JE}$ was performed on T-10 at RRC "Kurchatov Institute" in Moscow. 30 scientists from 13 countries participated in this experiment. The experiment was aimed to continue JE1 turbulence studies, now extending them to the plasma core.

The edge plasma in small and large scale experiments has many similar features. This has been demonstrated at the $2^{\text {nd }} \mathrm{JE}$ on $\mathrm{T}-10\left(\mathrm{R}=1.5 \mathrm{~m}, \mathrm{a}=36 \mathrm{~cm}, \mathrm{~B}_{\mathrm{t}}=2.5 \mathrm{~T}, \mathrm{I}_{\mathrm{pl}}=180\right.$ $330 \mathrm{kA}, \tau_{\text {pulse }}<1 \mathrm{~s}, n_{\mathrm{e}}=1.3-2.5 \times 10^{19} \mathrm{~m}^{-3}$ ) [4]. The absolute plasma potential was measured by Heavy Ion Beam probing (HIBP) [5] using $\mathrm{Tl}^{+}$ions with energy 220-260 keV. The core plasma turbulence was studied by correlation reflectometry. In the Ohmic plasma $\left(I_{\mathrm{p}}=180 \mathrm{kA}\right.$, $\left.n_{\mathrm{e}}=1.3-1.5 \times 10^{19} \mathrm{~m}^{-3}\right)$ the potential profile is a linear-like function with the lowest absolute value $\varphi(0.17 \mathrm{~m})=-900 \mathrm{~V}$. The slope of the potential profile gives the estimation of the mean radial electric field $E_{\mathrm{r}} \sim-7.5 \mathrm{kV} / \mathrm{m}$. In the ECR off-axis heated plasma $\left(P_{\mathrm{EC}}=0.4-0.8 \mathrm{MW}\right)$, the depth of the potential well becomes smaller, $\varphi(0.17 \mathrm{~m})=-720 \mathrm{~V}$, and the electric field decreases to $E_{\mathrm{r}} \sim-5.5 \mathrm{kV} / \mathrm{m}$. The turbulence rotation velocity obtained by correlation reflectometry [4] has been compared with this data, Fig.4, 5. 


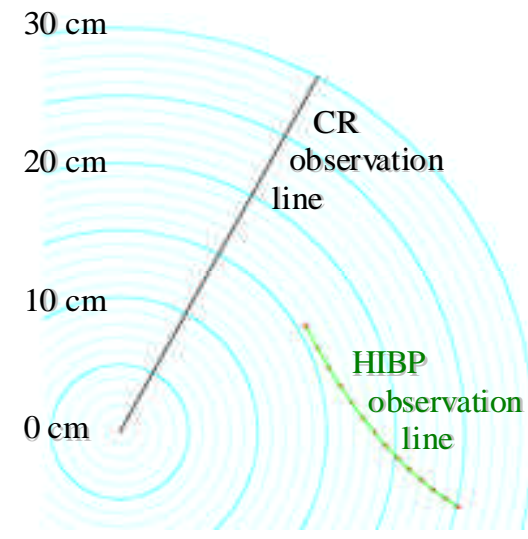

Fig. 4. Experimental layout for comparative rotation measurements.

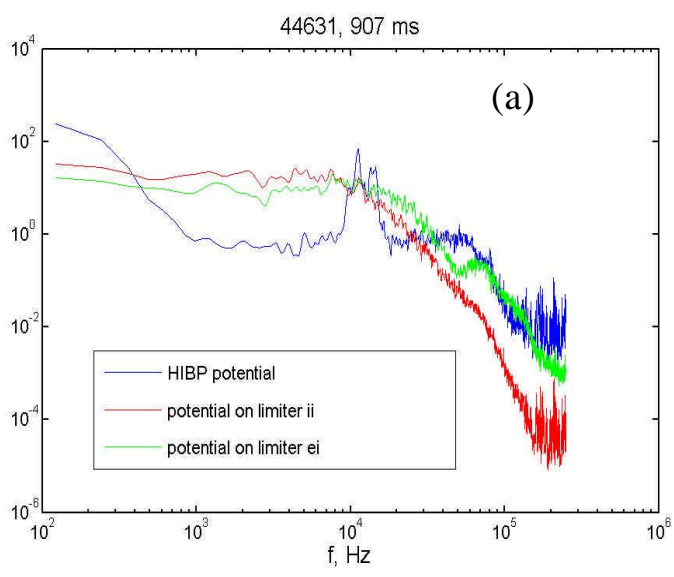

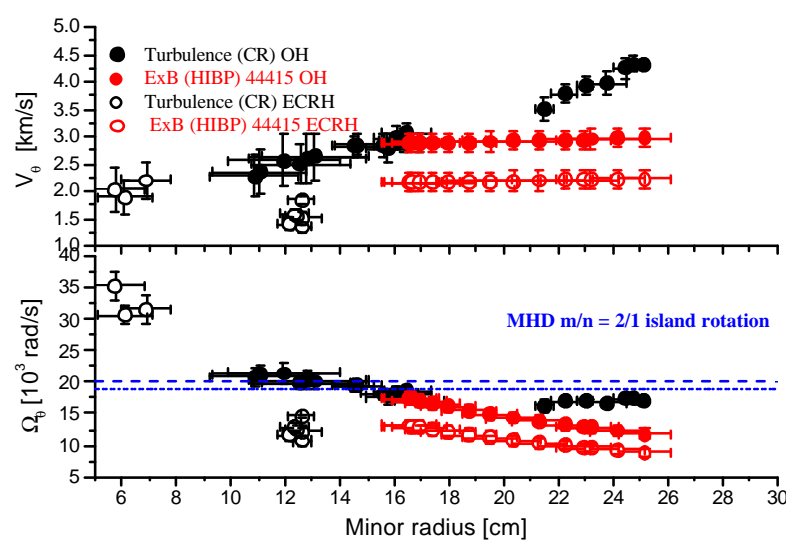

Fig. 5. Comparison of rotation velocities of density perturbations with core plasma rotation in $\mathrm{OH}$ and ECRH plasma.

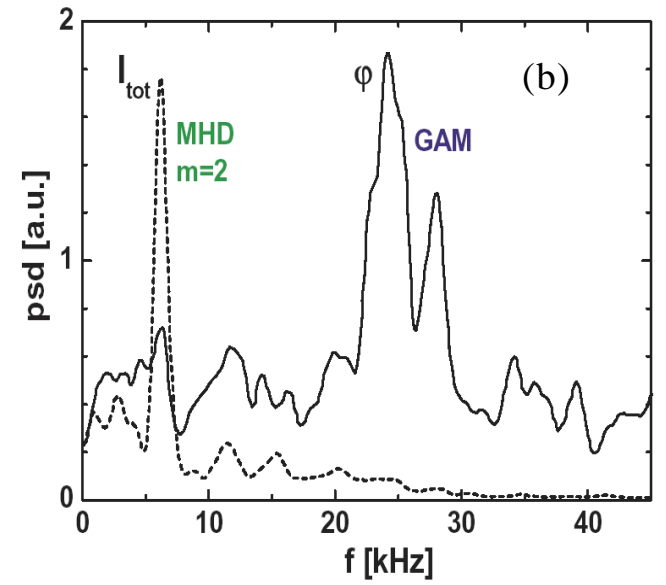

Fig 6. a) Potential power spectra in HIBP and limiter MLP, clear double peak at $20 \mathrm{kHz}$ characterize GAM, while no pronounced GAM peak in MPL spectra, b) Potential and $I_{\text {tot }}\left(n_{e}\right)$ spectra by HIBP.

During these experiments, a clear correlation between fluctuations in the plasma potential and density has been observed. Geodesic Acoustic Modes (GAMs) were investigated using the high energy ion beam diagnostics (HIBP), multipin movable and fixed limiter Langmuir probes (MLP) and correlation reflectometry (CR). Ohmic heating and onand off-axis ECRH regimes were studied $\left(\mathrm{B}_{\mathrm{t}}=2.2-2.5 \mathrm{~T}, \mathrm{I}_{\mathrm{pl}}=180-330 \mathrm{kA}, n_{\mathrm{e}}=1.3-\right.$ $\left.2.5 \times 10^{19} \mathrm{~m}^{-3}\right)$. The result of the correlation measurements of the two diagnostics allows determination of the toroidal and poloidal mode structure of the GAM. Figure 6 shows the potential and density power spectra, obtained by HIBP and MLP at the same time. It is clearly seen that the GAM peak is dominant in the potential spectra while an MHD $\mathrm{m}=2$ peak dominates the density spectra. It was shown that the GAM may have a complex structure, (not similar to conventional periodical oscillations with a single frequency), which is mainly manifested in the plasma potential and not very pronounced in plasma density fluctuations. 
The GAMs are more pronounced in the ECRH plasmas with typical frequencies of the wave packages in a narrow interval $22-27 \mathrm{kHz}$ in the outer one third region of the plasma column.

With a direct link to the experiments performed during the $1^{\text {st }} \mathrm{JE}$, studies of the edge plasma turbulence have been performed and the results have been compared with those

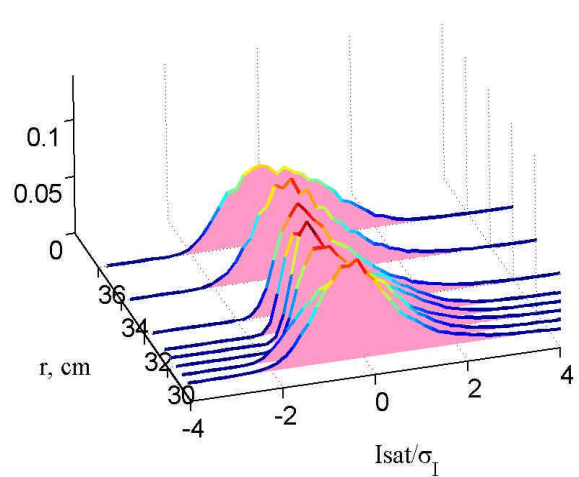

(a)

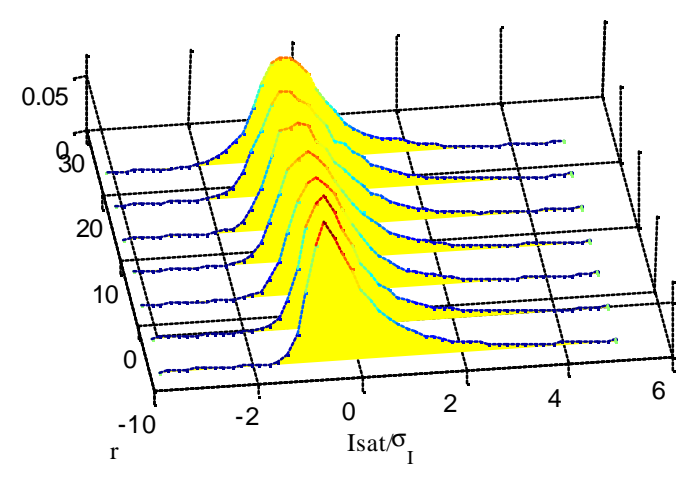

(b)

Fig.7. The PDF vs. radius in the SOL of T-10(a) and TCABR(b)

obtained on the TCABR tokamak in Brazil [4]. The analysis of probe signals has shown that the spectra, the correlation functions and the probability density functions (PDF), derived from probe ion saturation current, Fig.7, demonstrate complex power laws with multi-scale properties. Relative entropy and discrimination was used to compare PDF in T-10 and TCABR. The probability distribution function (PDF) varies with minor radius in both tokamaks, demonstrating evolution from strong non-gaussianity in the far SOL towards close to the Gaussian in the vicinity of the LCFS.

\section{The $3^{\text {rd }}$ JE, ISTTOK, ICT CFN, Lisbon, Portugal, 2007}

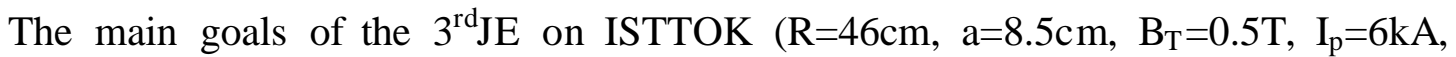
$\left.\overline{\mathrm{n}}_{\mathrm{e}}(0)=5 \times 10^{18} \mathrm{~m}^{-3}, \mathrm{~T}_{\mathrm{e}}(0)=150 \mathrm{eV}\right)$, were: tokamak operation in alternating current regimes; testing of the liquid metal limiter concept; study of the influence of external biasing on plasma confinement and stability and the study of fluctuation induced transport and assosiated driving mechanisms. The investigation of three-dimensional characteristics of the edge fluctuations has been performed with Langmuir probes showing that SOL fluctuations are characterized by short correlations both in space (poloidal) and time $\left(?_{\mathrm{c}} \sim 5-10 \mathrm{~mm}\right.$ and $\mathrm{t}_{\mathrm{c}}$ $\sim 5-8 \mu \mathrm{s}$, respectively), poloidal wave numbers in the range of $\mathrm{k}_{\text {? }}<3 \mathrm{~cm}^{-1}$, and a broad frequency spectrum, Fig.8. Deeper in the plasma the correlation is significantly larger $\left(?_{\mathrm{c}}>>10 \mathrm{~mm}, \mathrm{t}_{\mathrm{c}} \sim 30 \mu \mathrm{s}\right)$, the wave numbers are shorter $\left(\mathrm{k}_{?}<0.5 \mathrm{~cm}^{-1}\right)$ and the spectrum is dominated by low frequency components $(10-25 \mathrm{kHz})$. Similar to T-10, results suggest the existence of GAMs in a narrow region just inside the LCFS.

ISTTOK is equipped with a gallium jet injector which creates a jet $2.3 \mathrm{~mm}$ in diameter, $13 \mathrm{~cm}$ long with $2.5 \mathrm{~m} / \mathrm{s}$ flow velocity. The jet power extraction capability is extrapolated from the heat flux profiles. This work proved the technical feasibility of gallium jets interacting with plasmas. AC operations have also been investigated, with and without liquid metal limiter. Improvements in control systems have enabled achievement of $250 \mathrm{~ms}$ AC discharges, extending the plasma duration for almost one order of magnitude. Results from the HIBP diagnostics on T-10 and ISTTOK have been compared [6]. Other activities included familiarisation with the use of the ISTTOK control, data acquisition and remote data 
access systems; improvement of the remote access tools by adding new features based on user experience on ISTTOK and in other laboratories; implementation of a real-time Neural Network plasma position control system based on bolometer tomography, Fig.9; studies of plasma-material interaction. The development and implementation of remote participation tools is very important for supporting Joint Experiments and to allow remote collaboration. It is expected that the solutions implemented and being tested among the small tokamak community can provide useful experience for the development of a reliable standard supporting the future remote operation of large fusion devices. More results of this JE are presented in [7].

\section{Other activities within the IAEA CRP on RUST.}

Other activities within the IAEA CRP on RUST included co-operation and joint experiments on tokamaks participating in the $\mathrm{CRP}$ and also regular visits and consultations on the development of National Fusion programmes by the members of the CRP Scientific Committee. Joint Activity Matrix of collaboration between members of the CRP is shown in Table I. These activities resulted in more than 30 joint publications.

\section{Conclusions.}

The first Joint Experiments have clearly demonstrated that small tokamaks are suitable and important for broad international cooperation, providing the necessary environment and manpower to conduct dedicated joint research programmes. The contribution of small tokamaks to mainstream fusion research such as edge turbulence, improved confinement, and diagnostics development in the present case can be enhanced through coordinated planning. These activities under the IAEA CRP are already paying visible dividends and resulted in a substantial number of joint presentations and publications. The next Joint Experiment is scheduled for March 2009 on the TCABR tokamak at the University of São Paulo, Brazil to carry on investigation of correlations between the occurrence of transport barriers, improved confinement, electric fields and electrostatic turbulence.

Acknowledgements. Joint Experiments were carried out in the frame of the IAEA CRP of Research Using Small Tokamaks. This research has been partially supported by the grant KJB100430504 of Grant 
Agency of Academy of Sciences of the Czech Republic, by ROSATOM Goscontract RF 02.516.11.6068, RFBR 05-02-17016 and 07-02-01001, INTAS 100008-8046 and NWO-RFBR 047.016.015. Attendance was partially supported through the IAEA and ICTP grants.

Table I. Joint Activity Matrix of collaboration between members of the CRP.

\begin{tabular}{|c|c|c|c|c|c|c|c|c|c|c|c|c|c|}
\hline & \multicolumn{4}{|c|}{$\begin{array}{l}\text { 1. Core } \\
\text { transport and } \\
\text { turbulence }\end{array}$} & \multicolumn{3}{|c|}{$\begin{array}{l}\text { 2. Edge physics, } \\
\text { plasma surface } \\
\text { interaction and } \\
\text { technology }\end{array}$} & \multicolumn{3}{|c|}{$\begin{array}{l}\text { 3. Heating, current } \\
\text { drive and plasma } \\
\text { formation }\end{array}$} & \multicolumn{3}{|c|}{ 4. MHD and control } \\
\hline & \begin{tabular}{l|l}
$\exp$ & th \\
\end{tabular} & heory & tec & & exp & theory & tecl & exp & theory & tech & exp & theory & tech. \\
\hline $\mathbf{T}-10$ & $\mathbf{x}$ & $\mathbf{x}$ & & & $\mathbf{x}$ & $\mathbf{x}$ & $\mathbf{x}$ & & & & $\mathbf{x}$ & & $\mathbf{x}$ \\
\hline GUTTA & & & & & & & & $\mathbf{x}$ & & & $\mathbf{x}$ & $\mathbf{x}$ & \\
\hline SUNIST & & $\mathbf{x}$ & & & $\mathbf{x}$ & & & $\mathbf{x}$ & & $\mathbf{x}$ & & & \\
\hline EGYPTOR & & & & & $\mathbf{x}$ & & $\mathbf{x}$ & $\mathbf{x}$ & & $\mathbf{x}$ & $\mathbf{x}$ & & $\mathbf{x}$ \\
\hline ETE & & & & & $\mathbf{x}$ & & $\mathbf{x}$ & $\mathbf{x}$ & $\mathbf{x}$ & $\mathbf{x}$ & & $\mathbf{x}$ & \\
\hline TCABR & $\mathbf{x}$ & & & & $\mathbf{x}$ & & & $\mathbf{x}$ & $\mathbf{x}$ & $\mathbf{x}$ & $\mathbf{x}$ & & \\
\hline ISTTOK & & & & & $\mathbf{x}$ & & & $\mathbf{x}$ & $x$ & $\mathbf{x}$ & & & \\
\hline CASTOR & & & & & $\mathbf{x}$ & & & & & & & & \\
\hline STOR_M & $\mathbf{x}$ & $\mathbf{x}$ & & & $\mathbf{x}$ & & $\mathbf{x}$ & $\mathbf{x}$ & & $\mathbf{x}$ & $\mathbf{x}$ & $\mathbf{x}$ & \\
\hline IR-T1 & & & & & $\mathbf{x}$ & & & & & & $\mathbf{x}$ & $\mathbf{x}$ & \\
\hline TUMAN-3M & $\mathbf{x}$ & $\mathbf{x}$ & & & $\mathbf{x}$ & & & $\mathbf{x}$ & & & $\mathbf{x}$ & & \\
\hline \multirow[t]{3}{*}{ FT-2 } & $\mathbf{x}$ & $\mathbf{x}$ & & & $\mathbf{x}$ & & & $\mathbf{x}$ & & & $\mathbf{x}$ & & \\
\hline & \multicolumn{4}{|c|}{$\begin{array}{l}\text { 5. Diagnostics } \\
\text { development }\end{array}$} & \multicolumn{4}{|c|}{$\begin{array}{l}\text { 6. Control, data } \\
\text { acquisition, } \\
\text { remote } \\
\text { participation }\end{array}$} & \multicolumn{2}{|c|}{$\begin{array}{c}7 . \\
\text { Excellency } \\
\text { Education, } \\
\text { Capacity } \\
\text { Building } \\
\end{array}$} & $\begin{array}{c}8 . \\
\text { Expertise } \\
\text { exchange }\end{array}$ & \multicolumn{2}{|c|}{$\begin{array}{c}9 . \\
\text { Technology }\end{array}$} \\
\hline & exp & theo & & tech & & \begin{tabular}{l|l} 
exp & tr \\
\end{tabular} & neory & tech. & & & & & \\
\hline T-10 & $\mathbf{x}$ & & & $\mathbf{x}$ & & & & & $\mathbf{x}$ & & $\mathbf{x}$ & & $\mathbf{x}$ \\
\hline GUTTA & $\mathbf{x}$ & & & & & & & & $\mathbf{x}$ & & & & \\
\hline SUNIST & & & & & & & & & $\mathbf{x}$ & & $\mathbf{x}$ & & \\
\hline EGYPTOR & $\mathbf{x}$ & & & $\mathbf{x}$ & & & & $\mathbf{x}$ & & & $\mathbf{x}$ & & $\mathbf{x}$ \\
\hline ETE & $\mathbf{x}$ & $\mathbf{x}$ & & $\mathbf{x}$ & & & & & $\mathbf{x}$ & & $\mathbf{x}$ & & $\mathbf{x}$ \\
\hline TCABR & $\mathbf{x}$ & $\mathbf{x}$ & & $\mathbf{x}$ & & $\mathbf{x}$ & & $\mathbf{x}$ & $\mathbf{x}$ & & $\mathbf{x}$ & & \\
\hline ISTTOK & $\mathbf{x}$ & & & & & $\mathbf{x}$ & & $\mathbf{x}$ & $\mathbf{x}$ & & $\mathbf{x}$ & & \\
\hline CASTOR & $\mathbf{x}$ & $\mathbf{x}$ & & $\mathbf{x}$ & & & & $\mathbf{x}$ & $\mathbf{x}$ & & $\mathbf{x}$ & & $\mathbf{x}$ \\
\hline STOR_M & $\mathbf{x}$ & & & & & $\mathbf{x}$ & $\mathbf{x}$ & $\mathbf{x}$ & $\mathbf{x}$ & & $\mathbf{x}$ & & \\
\hline IR-T1 & $\mathbf{x}$ & & & $\mathbf{x}$ & & & & & $\mathbf{x}$ & & $\mathbf{x}$ & & \\
\hline TUMAN-3M & $\mathbf{x}$ & & & & & $\mathbf{x}$ & & & $\mathbf{x}$ & & $\mathbf{x}$ & & \\
\hline FT-2 & $\mathbf{x}$ & & & & & $\mathbf{x}$ & & & $\mathbf{x}$ & & $\mathbf{x}$ & & \\
\hline
\end{tabular}

[1] GRYAZNEVICH M P. et al., Nuclear Fusion 47 (2005) 269

[2] VAN OOST G. et al, Nuclear Fusion 47 (2007) 378

[3] VAN OOST G. et alPPCF, 45 (2003) 621, and references therein.

[4] GRYAZNEVICHM P et al., 34 ${ }^{\text {th }}$ EPS Conference on Pl. Phys. \& Controlled Fusion, Warsaw, Poland, P-1.070 (2007), and references therein.

[5] MELNIKOV A.V. et al., $32^{\text {nd }}$ EPS Conf. on Plasma Physics, Tarragona, P-4.089 (2005)

[6] VAN OOST G. et al, $35^{\text {th }}$ EPS Conference on Pl. Phys. \& Controlled Fusion, Crete, Greece, P-2.040 (2008)

[7] SILVA C. et al, this conference 